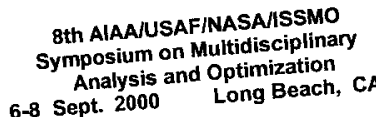

\title{
A00-40024
}

\section{TOPOLOGY OPTIMIZATION BASED ON STRUCTURAL FLEXIBILITY IN THE PERIODIC LOADING ${ }^{1}$}

\author{
Seungjae Min (seungjae@email.hanyang.ac.kr) \\ School of Mechanical Engineering, Hanyang University \\ 17 Haengdang-dong Sungdong-ku, Seoul 133-791, Korea \\ Shinji Nishiwaki (shinji@ket.tytlabs.co.jp) \\ Toyota Central R\&D Labs, Inc. \\ Nagakute Aichi, 480-1192, Japan \\ Noboru Kikuchi (kikuchi@engin.umich.edu) \\ Dept. of Mechanical Engineering \& Applied Mechanics, University of Michigan \\ Ann Arbor, MI 48109, USA
}

\begin{abstract}
In structural design, the stiffest structure has been considered optimal. However, if the flexibility is implemented in some appropriate portions, a flexible structure provides higher performance than the stiffest structure. Furthermore, since flexible portions can provide a mechanical function to the structure, a new breed of jointless structural mechanisms known as compliant mechanism can be designed with structural flexibility. In this study, we present a methodology to provide the optimal structure accounting for structural flexibility in the case of periodic input loads. First, dynamic mutual mean compliance is introduced in order to define flexibility using a mutual energy concept. Second, a new multi-objective function incorporating the maximization of flexibility and stiffness is proposed. Next, an optimization procedure is constructed based on the homogenization design method and sequential linear programming (SLP). Finally, some examples are presented to confirm the problem specifications of the optimal configurations.
\end{abstract}

\section{INTRODUCTION}

Topology optimization has been extensively considered to design the structural configuration for the stiffness maximization and the eigen-frequency maximization. We shall discuss a structural optimization method which implements structural flexibility in the case where structures are subjected to a periodic applied force in time using the homogenization design method. In general structural design, the stiffest structure has been considered optimal. However, by implementing flexibility in appropriate portions of the structure, we can obtain additional functions such as the mechanical function. A typical example of a structure with an additional mechanical function is a compliant mechanism (a flexible structure). It is designed to be flexible in order to achieve a specified motion.

The earliest effort of incorporating flexibility into a structure was made by Burns and Crossley ${ }^{1}$, and Midha and his associates ${ }^{2}$ developed a design method based on traditional rigid body. On the other hand, Ananthasuresh et al. ${ }^{3}$, Sigumud ${ }^{4}$, and Nishiwaki et al. ${ }^{5}$ constructed design methods based on the topology optimization technique. However, these works focus on the design of compliant mechanisms for static force input and do not consider the situation where compliant mechanisms are subjected to periodic loads in time.

It is also noted that we can design a high frequency actuator or a mechanical transducer by combining a flexible structure with excitation devices such as piezoceramic or electromagnetic devices (e.g. Onituska et al. ${ }^{6}$ ). Since these devices have small time constants, they can provide high frequency excitation. One drawback of such devices is that the output displacement is generally too small for use in an actuator or mechanical transducer. However, combining these devices with flexible structures can overcome this problem. That is, the mechanical structure can amplify the device displacement using flexibility. Typical applications are the design of the ultrasonic motor and the ultrasonic traveling wave linear motor. While several methods have been presented for analysis of this type of high frequency actuator using FEM (e.g. see Kagawa et al. ${ }^{7}$ ), a design method of a flexible structure for high frequency actuators has not been established.

' Copyright (C) 2000 by S. Min, S. Nishiwaki and N.

Kikuchi. Published by the American Institute of

Aeronautics and Astronautics, Inc. with permission. 
In this paper, we shall develop a methodology in which the homogenization design method is used to obtain the optimal structure design considering flexibility for cases in which the boundary is under a periodic load in time.

\section{HOMOGENIZATION DESIGN METHOD}

The homogenization design method has been widely used as a topology optimization method since Bendsøe and $\mathrm{Kikuchi}^{8}$ introduced. The key ideas are the use of the extended and fixed design domain $D$ which includes the original design domain $\Omega_{d}$, a priori, and the introduction of the characteristic function defined by

$$
\chi_{\Omega}(x)= \begin{cases}1 & \text { if } x \in \Omega_{d} \\ 0 & \text { if } x \in D \backslash \Omega_{d}\end{cases}
$$

This characteristic function allows to describe any shape or topology as an optimal configuration.

Since the characteristic function can have only the discretized value, either 0 or 1 in an infinitely small area, the physical properties defined using the characteristic function are very discontinuous everywhere in the extended design domain $D$. This nature makes numerical treatment of the structural optimization problem difficult. To overcome this problem, Bendsøe and Kikuchi $^{8}$ utilized the homogenization method. In this method, the discontinuous physical properties are relaxed to smooth functions as the homogenized properties. The extended design domain $D$ is reconfigured using the homogenized properties.

Consider the microstructures shown in Fig. 1. The microstructure is formed inside an empty rectangle in a unit cell, where $\alpha, \beta$, and $\theta$ are regarded as the design variables. The variable $\theta$ represents the rotation of the unit cell.

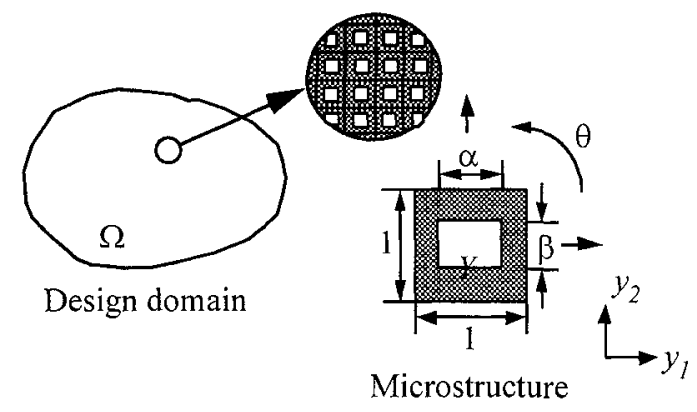

Fig. 1. Microstructures for the relaxation of the design domain

First, we calculate the homogenized elasticity tensor, $E^{H}$, in the case where the angle $\theta$ is set to 0 . To obtain this homogenized elasticity tensor, the characteristic deformations, $\chi(x, y)$, are calculated using the following equation:

$$
\begin{aligned}
& \int_{Y} \varepsilon_{y}(v)^{T} E(x, y) \varepsilon_{y}(\chi(x, y)) d Y \\
& =\int_{Y} \varepsilon_{y}(v)^{T} E(x, y) d Y \text { for } \forall v \in V_{y}
\end{aligned}
$$

where $\varepsilon_{y}(v)^{T}=\left\{\frac{\partial v_{1}}{\partial y_{1}} \frac{\partial v_{2}}{\partial y_{2}} \frac{1}{2}\left(\frac{\partial v_{1}}{\partial y_{2}}+\frac{\partial v_{2}}{\partial y_{1}}\right)\right\} \quad$ and $V_{y}$ is the admissible space defined in the unit cell $\boldsymbol{Y}$ such that $V_{y}=\left\{v: v_{i} \in H^{1}(Y) \mid v\right.$ is Y-periodic $\}$. After obtaining the characteristic deformations, the homogenized elasticity tensor, $E^{H}$, is computed by

$$
E^{H}=\frac{1}{|Y|} \int_{Y} E(x, y)\left(I-\varepsilon_{y}(\chi)\right) d Y
$$

where $|Y|$ stands for the area of the unit cell. Next, when the unit cell is rotated by angle $\theta$, the homogenized elasticity tensor, $E^{G}$, is computed by

$$
E^{G}=R(\theta)^{T} E^{H} R(\theta)
$$

where $\boldsymbol{R}$ is the rotation matrix.

Thus, the homogenized elasticity tensor, $E^{G}$, is determined by the microscopic design variables $\alpha, \beta$, and $\theta$.

\section{FORMULATION OF FLEXIBILITY AND STIFFNESS UNDER PERIODIC LOADS}

The flexibility and stiffness are formulated in the case where the periodic loads in time are applied using the mutual energy concept introduced by Shield and Prager $^{9}$ and Huang ${ }^{10}$. Suppose that an elastic body occupying a two dimensional domain, $\Omega$, is fixed at boundary $\Gamma_{d}$. Now we consider the two equilibrium cases with a different traction in a different boundary: Case (a) and Case (b), as shown in Fig. 2. That is, in Case (a), the elastic body is subjected to boundary traction $t^{1}$ at boundary $\Gamma_{t^{\prime}}$, and in Case (b), it is subjected to boundary traction $t^{2}$ at boundary $\Gamma_{t^{2}}$. Body forces applied to the elastic body and the damping effect are assumed to be ignored for simplicity in the formulation. The displacement fields are $u^{1}=\left\{u_{1}^{1}, u_{2}^{1}\right\}$ in Case (a), and $u^{2}=\left\{u_{1}^{2}, u_{2}^{2}\right\}$ in Case (b). We suppose that tractions and are harmonic excitations to the elastic body, and displacement fields and are also harmonic in the steady state. That is, tractions $t^{1}$ and $t^{2}$ and displacement fields $u^{1}$ and $u^{2}$ are assumed to be described as

$$
\begin{aligned}
t^{1} & =T^{1} e^{j \omega t}, t^{2}=T^{2} e^{j \omega t} \\
u^{1} & =U^{1} e^{j \omega t}, u^{2}=U^{2} e^{j \omega t}
\end{aligned}
$$

where $\omega$ stands for an excitation frequency, $t$ stands for time, and $T^{1}, T^{2}, U^{1}$ and $U^{2}$ stand for amplitudes of $t^{3}, t^{2}, u^{1}$ and $u^{2}$, respectively. 
Here, we introduce the linear form implying the mutual mean compliance in the dynamic problem defined by

$$
L^{2}\left(U^{1}\right)=\int_{\Gamma^{2}} T^{2} U^{1} d \Gamma=\int_{\Gamma_{, 2}} T_{i}^{2} U_{i}^{1} d \Gamma, U^{1} \in V
$$

where $V$ is the admissible linear space such that $V=\left\{v=v_{i} e_{i}: v_{i} \in H^{1}(\Omega)\right.$ with $v=0$ on $\left.\Gamma_{d}, i=1,2\right\}$.

We assume that boundary traction $t^{1}$ in Case (a) is an applied force, and the amplitude of boundary traction $T^{2}$ in Case (b) is a unit dummy vector. Then, mutual mean compliance $L^{2}\left(U^{1}\right)$ represents the measure of deformation at boundary $\Gamma_{t^{2}}$ when we apply boundary traction $t^{i}$ at boundary $\Gamma_{t^{2}}$. That is, mutual mean compliance $L^{2}\left(U^{1}\right)$ is interpreted as how flexible or stiff boundary $\Gamma_{t^{2}}$ is when boundary traction $t^{\prime}$ is applied at boundary $\Gamma_{t^{\prime}}$ in the dynamic case. Therefore, by maximizing or increasing the absolute value of $L^{2}\left(U^{1}\right)$, we can obtain sufficient flexibility along a direction specified by $T^{2}$ with respect to $t^{1}$. Note that both the maximization of $L^{2}\left(U^{1}\right)$ and minimization of $L^{2}\left(U^{1}\right)$ provide sufficient flexibility, because under harmonic excitation, the deformation in the direction of $-T^{2}$, where the phase angle is $\omega t$, is identical to the deformation in the direction of $T^{2}$, where the phase angle is $\omega t+\pi$.
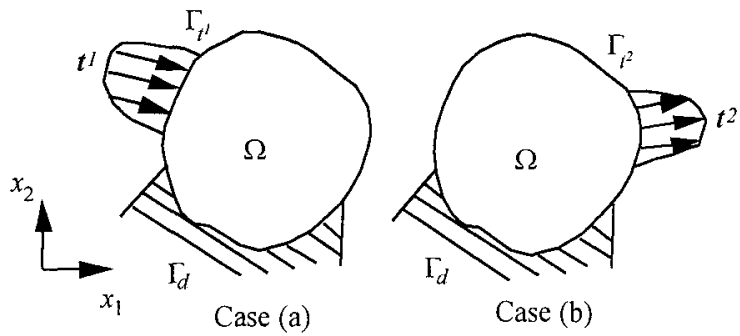

Fig. 2. An elastic body subjected to two tractions

The following bilinear forms to describe the equilibrium equations are introduced by the concept of mutual potential energy:

$$
\begin{aligned}
& a_{E}\left(U^{1}, v^{2}\right)-\omega^{2} a_{M}\left(U^{1}, v^{2}\right)=L^{1}\left(v^{2}\right) \\
& \text { for } U^{1} \in V, \forall v^{2} \in V \\
& a_{E}\left(U^{2}, v^{1}\right)-\omega^{2} a_{M}\left(U^{2}, v^{1}\right)=L^{2}\left(v^{1}\right) \\
& \text { for } U^{2} \in V, \forall v^{1} \in V \\
& a_{E}(u, v)=\int_{\Omega} \varepsilon(v)^{T} E \varepsilon(u) d \Omega \\
& \qquad a_{M}(u, v)=\int_{\Omega} \rho u v d \Omega
\end{aligned}
$$

with linearized strains

$$
\varepsilon(u)=\varepsilon_{i j}(u)=\frac{1}{2}\left(\frac{\partial u_{i}}{\partial x_{j}}+\frac{\partial u_{j}}{\partial x_{i}}\right)
$$

where $E$ is the elasticity tensor and $\rho$ is the mass density. Substituting $U^{2}$ into $v^{2}$ in (8) and $U^{1}$ into $v^{1}$ in (9), we obtain the following relation at equilibrium:

$$
L^{1}\left(U^{2}\right)=a_{E}\left(U^{1}, U^{2}\right)-\omega^{2} a_{M}\left(U^{1}, U^{2}\right)=L^{2}\left(U^{1}\right)(
$$

and the total mutual potential energy can be written as follows:

$$
\begin{aligned}
& F\left(U^{1}, U^{2}\right)=\frac{1}{2} a_{E}\left(U^{1}, U^{2}\right)-\frac{1}{2} \omega^{2} a_{M}\left(U^{1}, U^{2}\right) \\
& -\frac{1}{2} L^{1}\left(U^{2}\right)-\frac{1}{2} L^{2}\left(U^{1}\right)=-\frac{1}{2} L^{\mathrm{l}}\left(U^{2}\right)=-\frac{1}{2} L^{2}\left(U^{1}\right)
\end{aligned}
$$

Taking the first variation of $F$ with respect to $U^{1}$, $U^{2}$ and a design variable $A$ yields

$$
\begin{aligned}
& \delta F\left(U^{1}, U^{2}\right)=\frac{1}{2} a_{E}\left(U^{2}, \frac{\partial U^{1}}{\partial A} \delta A+\delta U^{1}\right) \\
& -\frac{1}{2} \omega^{2} a_{M}\left(U^{2}, \frac{\partial U^{1}}{\partial A} \delta A+\delta U^{1}\right) \\
& -\frac{1}{2} L^{2}\left(\frac{\partial U^{1}}{\partial A} \delta A+\delta U^{1}\right) \\
& +\frac{1}{2} a_{E}\left(U^{1}, \frac{\partial U^{2}}{\partial A} \delta A+\delta U^{2}\right) \\
& -\frac{1}{2} \omega^{2} a_{M}\left(U^{1}, \frac{\partial U^{2}}{\partial A} \delta A+\delta U^{2}\right) \\
& -\frac{1}{2} L^{1}\left(\frac{\partial U^{2}}{\partial A} \delta A+\delta U^{2}\right) \\
& +\frac{1}{2} \zeta_{\Omega} \varepsilon\left(U^{2}\right)^{T} \frac{\partial E}{\partial A} \varepsilon\left(U^{1}\right) d \Omega \delta A \\
& -\frac{1}{2} \omega^{2} J_{\Omega} \frac{\partial \rho}{\partial A} U^{1} \cdot U^{2} d \Omega \delta A
\end{aligned}
$$

Using equilibrium equations (8), (9) and (14), the sensitivities of the total mutual potential energy and the mutual mean compliance $L^{2}\left(U^{1}\right)$ with respect to a design variable $A$ yield, respectively,

$$
\begin{aligned}
& \frac{\partial F}{\partial A}=\frac{1}{2} \int_{\Omega} \varepsilon\left(U^{2}\right)^{T} \frac{\partial E}{\partial A} \varepsilon\left(U^{1}\right) d \Omega \\
& -\frac{1}{2} \omega^{2} \int_{\Omega} \frac{\partial \rho}{\partial A} U^{1} \cdot U^{2} d \Omega
\end{aligned}
$$

and

$$
\begin{aligned}
& \frac{\partial L^{2}\left(U^{1}\right)}{\partial A}=-\int_{\Omega} \varepsilon\left(U^{2}\right)^{T} \frac{\partial E}{\partial A} \varepsilon\left(U^{1}\right) d \Omega \\
& +\omega^{2} \int_{\Omega} \frac{\partial \rho}{\partial A} U^{1} \cdot U^{2} d \Omega
\end{aligned}
$$

Next, we only consider Case (a). The linear form implying the mean compliance in the dynamic problem is defined by 


$$
L^{1}\left(U^{1}\right)=\int_{\Gamma_{i}^{2}} T^{1} U^{1} d \Gamma=\int_{r_{i}^{1}} T_{i}^{1} U_{i}^{1} d \Gamma, U^{1} \in V
$$

The mean compliance $L^{1}\left(U^{1}\right)$ is interpreted as the measure of stiffness at boundary $\Gamma_{t^{\prime}}$ when we apply boundary traction $t^{1}$ at boundary $\Gamma_{t^{\prime}}$. By minimizing or decreasing the absolute value of $L^{l}\left(U^{\prime}\right)$, we can obtain sufficient stiffiness along a direction specified by $T^{1}$. Since the displacement fields satisfy the following the equilibrium equations:

$$
\begin{aligned}
& a_{E}\left(U^{1}, v^{1}\right)-\omega^{2} a_{M}\left(U^{1}, v^{1}\right)=L^{1}\left(v^{1}\right) \\
& \text { for } U^{1} \in V, \forall v^{1} \in V
\end{aligned}
$$

we obtain the following relations at equilibrium:

$$
\begin{gathered}
a_{E}\left(U^{1}, U^{1}\right)-\omega^{2} a_{M}\left(U^{1}, U^{1}\right)=L^{1}\left(U^{1}\right) \\
F\left(U^{1}, U^{1}\right)=\frac{1}{2} a_{E}\left(U^{1}, U^{1}\right)-\frac{1}{2} \omega^{2} a_{M}\left(U^{1}, U^{1}\right) \\
-L^{1}\left(U^{1}\right)=-\frac{1}{2} L^{1}\left(U^{1}\right)
\end{gathered}
$$

Taking the first variation of $F$ with respect to $U^{1}$ and a design variable $A$ and using equilibrium equations (20) and (21), the sensitivities of the total mutual potential energy and the mutual mean compliance $L^{1}\left(U^{1}\right)$ with respect to a design variable $A$ yield, respectively,

$$
\begin{aligned}
& \frac{\partial F}{\partial A}=\frac{1}{2} \int_{\Omega} \varepsilon\left(U^{1}\right)^{T} \frac{\partial E}{\partial A} \varepsilon\left(U^{1}\right) d \Omega \\
& -\frac{1}{2} \omega^{2} \int_{\Omega} \frac{\partial \rho}{\partial A} U^{1} \cdot U^{1} d \Omega
\end{aligned}
$$

and

$$
\begin{aligned}
& \frac{\partial L^{1}\left(U^{1}\right)}{\partial A}=-\int_{\Omega} \varepsilon\left(U^{1}\right)^{T} \frac{\partial E}{\partial A} \varepsilon\left(U^{1}\right) d \Omega \\
& +\omega^{2} \int_{\Omega} \frac{\partial \rho}{\partial A} U^{1} \cdot U^{1} d \Omega
\end{aligned}
$$

\section{FORMULATION OF THE MULTI-OBJECTIVE OPTIMIZATION PROBLEM}

Suppose that the original design domain $\Omega_{d}$ of a flexible structure is fixed at boundary $\Gamma_{d}$ and is subjected to the periodically oscillating boundary traction $t^{1}=T^{1} e^{j \omega t}$, where $\omega$ is an excitation frequency and $t$ is time, as shown in Fig. 3.
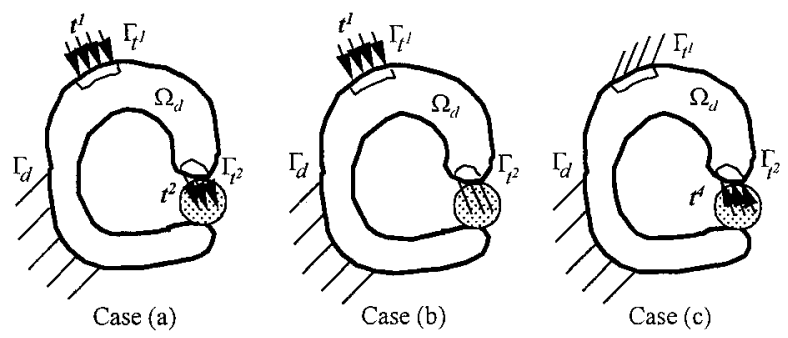
posed by the reaction force of the reaction force while boundary $\Gamma_{t^{\prime}}$ is fixed because the flexible structure must be imposed by applied traction $t^{1}$. The direction of the reaction force is assumed to be opposite to that of dummy load vector $T^{2}$. However, the excitation frequency of the reaction force is not uniquely determined, a priori, and is usually unknown in the design phase of some flexible structures, since this depends on the contact condition. In this case, we assume that the reaction force is considered to be statically applied in the engineering sense, and the problem can be formulated as follows:

Case (c)

$$
\min _{\alpha, \beta, \theta}\left|L^{4}\left(u^{4}\right)\right|=\left|\int_{\Gamma_{, 2}} T^{4} u^{4} d \Gamma\right|
$$

subject to

$$
\begin{gathered}
T^{4}=-T^{2} \\
a_{E}\left(U^{4}, v^{4}\right)-\omega_{R}{ }^{2} a_{M}\left(U^{4}, v^{4}\right)=L^{4}\left(v^{4}\right) \\
\text { for } u^{4} \in V^{(c)}, \forall v^{4} \in V^{(c)} \\
V^{(c)}=\left\{\begin{array}{l}
v: v_{i} \in H^{1}\left(\Omega_{d}\right) \\
\text { with } v=0 \text { on } \Gamma_{d} \text { and } \Gamma_{t^{1}}
\end{array}\right\} \\
g(\alpha, \beta)=\int_{\Omega_{d}}(1-\alpha \beta) d \Omega-\Omega_{s} \leq 0
\end{gathered}
$$

where $\omega_{R}$ is the excitation frequency of the reaction force.

To satisfy the kinematic and the stiffness requirements, it is necessary to consider three optimization problems and the multi-objective optimization problem is formulated to design a flexible structure under a periodic load in time. The weighting method has been employed most commonly because of the simplicity, but in this problem, the mutual mean compliance $L^{2}\left(U^{1}\right)$ can be close to infinite with certain weighting coefficients. A different form of a multi-objective function is proposed to find the appropriate optimal configuration in the Pareto optima as follows:

$$
\begin{aligned}
& \max _{\alpha, \beta, \theta} f=W \log \left|L^{2}\left(U^{1}\right)\right|- \\
& \quad \frac{1}{2}(1-W) \log \left[w_{s} L^{3}\left(U^{3}\right)^{2}+\left(1-w_{s}\right) L^{4}\left(U^{4}\right)^{2}\right]
\end{aligned}
$$

where $W$ and $w_{s}$ are the weighting coefficients such that $0 \leq W \leq 1$ and $0 \leq w_{s} \leq 1 . W$ represents the relative importance of flexibility and stiffness. $w_{s}$ represents the relative importance of two stiffnesses defined in Case (b) and Case (c) shown in Fig. 3. We take squares of two mean compliances $L^{3}\left(u^{3}\right)$ and $L^{4}\left(u^{4}\right)$ since the absolute values of them must be minimized to obtain sufficient stiffness in the dynamics case. It is noted that since weighting coefficient $W$ depends on excitation frequency $\omega$, we must adjust this weighting coefficient for a different frequency problem. Since the multiobjective function is composed of the logarithm functions, the optimal solution obtained is one of the Pareto optimas.

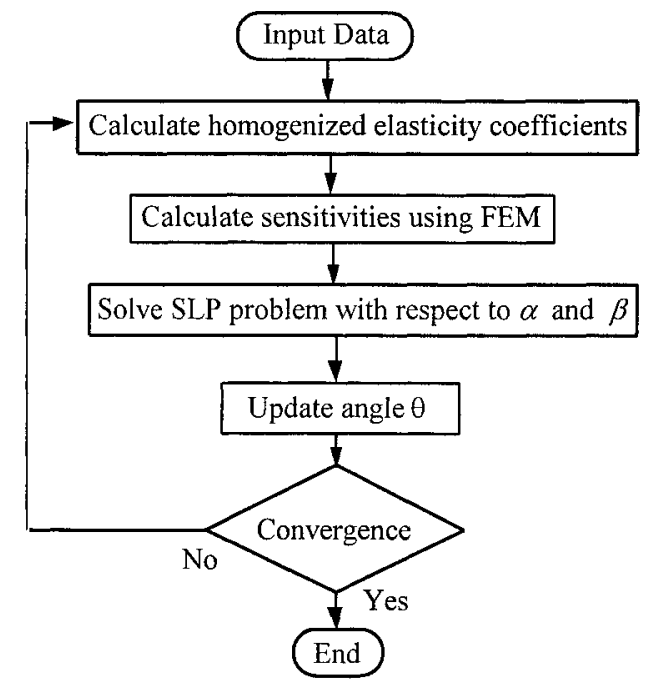

Fig. 4. Optimization procedure

Fig. 4 shows a flowchart of the optimization procedure. In the first step, homogenized elasticity tensor is computed using the finite element method Using the finite element method, the numerical values of homogenized elasticity tensor are calculated. In the second step, the mutual mean compliance, the two mean compliances, the total volume, and the objective function are computed using FEM. Extended design domain $D$ is discretized by the finite elements. We approximate that the configuration of the microstructure is uniform in each element. The configuration of the microstructure in the $i$-th element can be represented by three design variables, $\alpha_{i}, \beta_{i}$, and $\theta_{i}$ for $i=1, \ldots, n$, where $n$ is the number of element. Therefore, the total number of design variables is $3 n$ in the entire design domain $D$. In the third step, sensitivities of mutual compliance, the two mean compliances, and total volume, and the objective function with respect to design variables are computed if the objective function is not converged. In the fourth step, the optimization problem with $\alpha_{i}$ and $\beta_{i}$ is solved by sequential linear programming (SLP). SLP can deal with a variety of objective functions and can handle numerous design variables although fast convergence cannot be expected. In the fifth step, angle $\theta_{i}$ is updated. In this study, this angle is practically updated to minimize the two mean compliances for simplicity of computation. To minimize the two mean compliances, the direction of angle $\theta_{i}$ is updated to the principal direction of stress using the multi-loading criterion. 


\section{NUMERICAL EXAMPLES}

Fig. 5 shows the design domain where boundary conditions and specifications are as indicated. The deformation along a direction specified by dummy load at B is to be maximized when the periodically oscillating force along a direction specified at point $A$, while the stiffnesses at both points $A$ and $B$ are to be maximized. Points $\mathrm{A}$ and $\mathrm{B}$ are corresponding to $\Gamma_{t^{1}}$ and $\Gamma_{t^{2}}$, respectively. The properties of the isotropic material correspond to Young's modulus $=$ 100 , Poisson's ratio $=0.3$, and mass density $=7.8510^{\circ}$ ${ }^{6}$. The magnitude of amplitude of an applied force is assumed to be a unit load. The design domain is discretized using 1800 QUAD4 finite elements. The total volume constraint of the material $\Omega_{s}$ is considered to be $20 \%$ of the volume of the whole design domain.

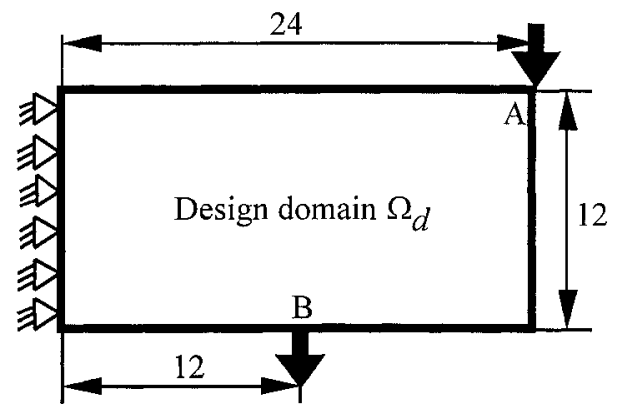

Fig. 5. Design domain for simple model

\section{- Optimal solutions with different weighting coefficient $W$}

There exist many local optima in this dynamic problem since we can achieve the same eigenfrequency with different material distributions in the fixed design domain in order to obtain sufficient flexibility. We must choose appropriate initial configurations in order to obtain the appropriate optimal configurations which have physical meaning among local optima. Therefore, we basically use the optimal configuration in the static case as an initial configuration in the dynamic case. Fig. 6 shows the optimal configuration in the case of excitation frequency $\omega=0(\mathrm{rad} / \mathrm{s})(0 \mathrm{~Hz})$ and $w_{s}=0.4$ using the uniform initial configuration where values of microscopic design variables $\alpha_{i}$ and $\beta_{i}$ are set to 0.90 , and the value of $\theta_{i}$ is set to 0.0 for all elements. To investigate the effect of the weighting coefficient $W$, Fig. 6 is utilized as an initial configuration while the excitation frequency is set to $\omega=502.65(\mathrm{rad} / \mathrm{s})$ $(80 \mathrm{~Hz})$. Table 1 shows values of three objective functions and the lowest eigen-frequencies of optimal configurations with different weighting coefficients $W$, and we can see that each optimal configuration has the lowest eigen-frequency which is almost the same as the excitation frequency of the applied force. It is reasonable that the resonance situation can provide the highest flexibility if we do not take into account the structural requirement. However, due to the structural requirement which prevents the objective function from going toward infinity, the lowest eigen-frequency is not exactly the same as the excitation frequency of the applied force. Fig.7 and Fig.8 show the optimal configuration and its eigenmode of the lowest eigen-frequency, respectively, in the case of $W=0.02$.

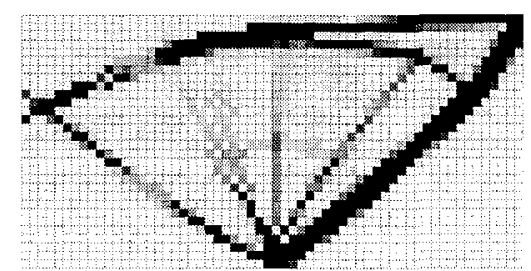

Fig. 6. Optimal configuration

$$
\left(\omega=0(0 \mathrm{~Hz}), W=0.5, w_{s}=0.4\right)
$$

Table 1. Objective functions at optimal points (Optimal configurations in Fig. 6 used as initail solutions)

$$
\omega=502.65(\mathrm{rad} / \mathrm{s})(80 \mathrm{~Hz}), w_{s}=0.4
$$

\begin{tabular}{|r|r|r|r|r|}
\hline$W$ & case (a) & case (b) & case (c) & Eigen-frequency $(\mathrm{Hz}$ \\
\hline 0.01 & 6.811 & 0.359 & 0.147 & 32.78 \\
\hline 0.02 & 1146.86 & 0.362 & 0.149 & 79.81 \\
\hline 0.03 & 1450.96 & 0.374 & 0.153 & 80.17 \\
\hline 0.05 & 290.52 & 0.390 & 0.156 & 80.90 \\
\hline 0.08 & 432.96 & 0.402 & 0.161 & 80.62 \\
\hline 0.1 & 421.51 & 0.417 & 0.163 & 80.80 \\
\hline 0.3 & 713.03 & 0.535 & 0.180 & 79.24 \\
\hline 0.5 & 198.041 & 0.633 & 0.198 & 80.81 \\
\hline
\end{tabular}

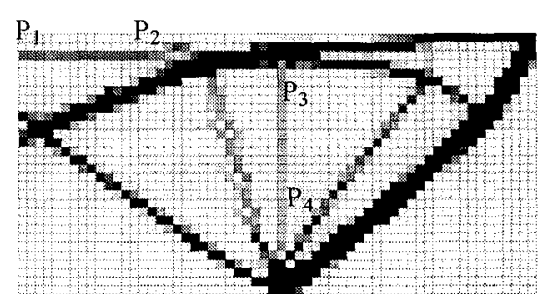

Fig. 7. Optimal configuration $\left(\omega=502.65(80 \mathrm{~Hz}), W=0.02, w_{\mathrm{s}}=0.4\right)$

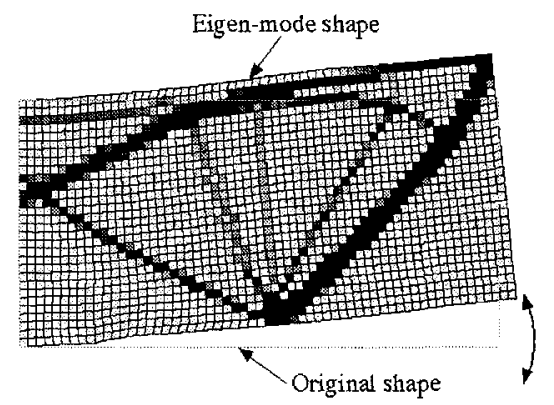

Fig. 8. Eigen-mode of the lowest eigen-frequency

- Optimal solutions with different weighting coefficient $w_{s}$

Table 2 shows values of three objective functions and the lowest eigen-frequencies of optimal configurations with different weighting coefficients 
$w_{s}$ while Fig.6 is also utilized as an initial configuration and $W$ is set to 0.02 . It is noted that the weighting coefficients $w_{s}$ corresponding to the relative stiffness significantly change the flexibility defined by the mutual mean compliance, but have little influences to the stiffness. Fig. 9 shows the optimal configuration in the case of $w_{s}=0.8$ and gives similar topology except the portion connecting from $P_{1}$ to $P_{2}$ compared with Fig. 7 .

Table 2. Objective functions at optimal points (Optimal configurations in Fig. 6 used as initail solutions) $\omega=502.65(\mathrm{rad} / \mathrm{s})(80 \mathrm{~Hz}), W=0.02$

\begin{tabular}{|r|r|r|r|r|}
\hline$w_{c}$ & case (a) & case (b) & case (c) & Eigen-frequency (Hz) \\
\hline 0.2 & 6.863 & 0.371 & 0.125 & 31.12 \\
\hline 0.4 & 1146.86 & 0.362 & 0.149 & 79.81 \\
\hline 0.6 & 739.45 & 0.363 & 0.168 & 79.71 \\
\hline 0.8 & 1779.13 & 0.363 & 0.213 & 79.93 \\
\hline
\end{tabular}

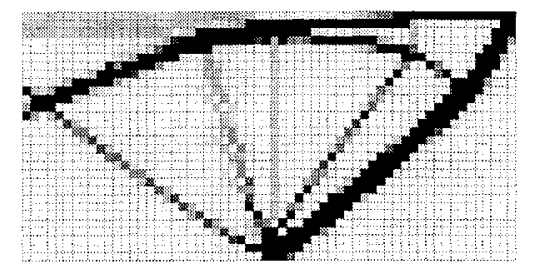

Fig. 9. Optimal configuration $\left(\omega=502.65(80 \mathrm{~Hz}), W=0.02, w_{s}=0.8\right)$

- Optimal solutions with different excitation frequency $\omega$

Table 3 summarizes values of three objective functions and the lowest eigen-frequencies of optimal configurations with different excitation frequencies $\omega$ while $w_{s}$ is set to 0.4. Different weighting coefficients $W$ and initial configurations are required to obtain physically meaningful structures. Fig.10 shows that there is little difference in structural topology up to $\omega=251.33(\mathrm{rad} / \mathrm{s})(40 \mathrm{~Hz})$. As the excitation frequency becomes higher than $40 \mathrm{~Hz}$, the structural portion connecting from $\mathrm{P}_{1}$ to $\mathrm{P}_{2}$ is generated, and on the contrary, no materials are distributed to the portion from $\mathrm{P}_{3}$ to $\mathrm{P}_{4}$.

It is clear that the optimal configuration depends on the excitation frequency and is noted that each optimal configuration has the lowest eigen-frequency which is almost the same as the excitation frequency of the applied force. That is, the dynamic effect affects the topology configuration, especially in the high excitation frequency case. Therefore, the dynamic effect must be considered in the design phase if the excitation frequency of the applied force is high.

Table 3. Relation between the applied frequency and objective functions $\left(w_{\mathrm{s}}=0.4\right)$

\begin{tabular}{|r|r|r|r|r|r|}
\hline$\omega / 2 \pi(\mathrm{Hz})$ & $W$ & case (a) & case (b) & case (c) & E.f.(Hz) \\
\hline 40 & 0.005 & 919.16 & 0.383 & 0.152 & 39.55 \\
\hline 80 & 0.02 & 1146.86 & 0.362 & 0.149 & 79.81 \\
\hline 120 & 0.05 & 509.54 & 0.372 & 0.156 & 119.62 \\
\hline 160 & 0.2 & 284.21 & 0.479 & 0.166 & 159.26 \\
\hline 200 & 0.1 & 191.97 & 0.434 & 0.168 & 199.32 \\
\hline 240 & 0.4 & 518.87 & 0.643 & 0.202 & 240.51 \\
\hline
\end{tabular}

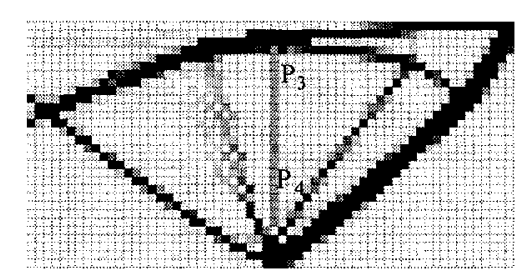

Fig. 10. Optimal configuration $\left(\omega=251.33(40 \mathrm{~Hz}), W=0.005, w_{\mathrm{s}}=0.4\right)$

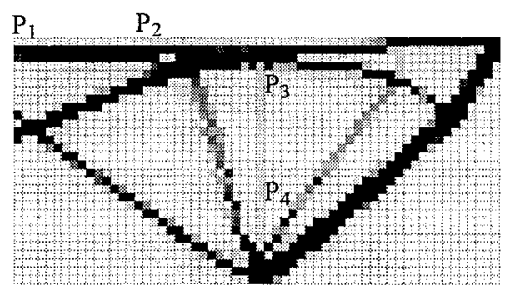

Fig. 11. Optimal configuration $\left(\omega=753.98(120 \mathrm{~Hz}), W=0.05, w_{s}=0.4\right)$

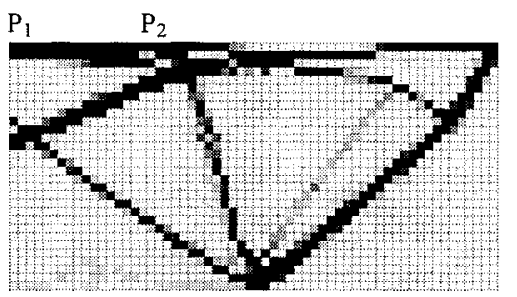

Fig. 12. Optimal configuration $\left(\omega=1005.31(160 \mathrm{~Hz}), W=0.2, w_{s}=0.4\right)$

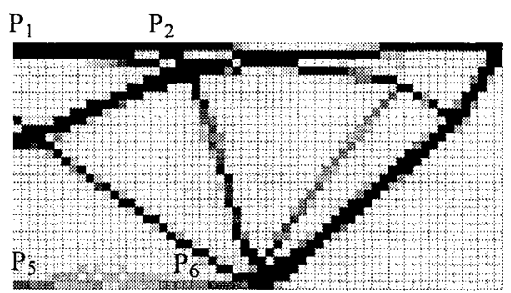

Fig. 13. Optimal configuration $\left(\omega=1256.64(200 \mathrm{~Hz}), W=0.1, w_{\mathrm{s}}=0.4\right)$

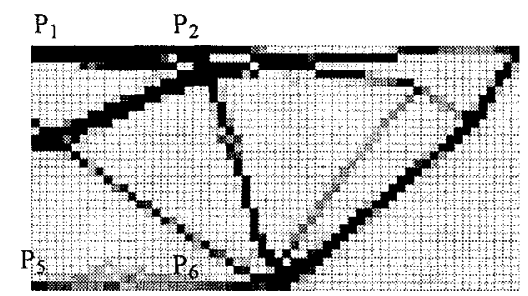

Fig. 14. Optimal configuration $\left(\omega=1507.96(240 \mathrm{~Hz}), W=0.4, w_{\mathrm{s}}=0.4\right)$ 


\section{CONCLUSIONS}

In this research, we developed a topology optimization method implementing flexibility for cases in which the boundary is under a periodic load in time. First, the mutual mean compliance was formulated using the mutual energy concept in order to define the measure of flexibility in the dynamic case. The sensitivity of the mutual mean compliance with respect to a design variable was derived. Second, the multi-objective optimization problem was formulated in order to design a flexible structure under a periodic load in time. A new multi-objective function was also formulated in order to obtain appropriate optimal solutions which have physical meaning. Next, the optimization algorithm was constructed based on the homogenization design method. Finally, two design examples were presented to examine the characteristics of the optimal configurations. These examples confirm that the flexible structure under a periodic load can be designed using the method presented here.

\section{ACKNOWLEDGEMENT}

This research was supported by Center of Innovative Design Optimization Technology (ERC of Korea Science and Engineering Foundation).

\section{REFERENCES}

(1) Burns, R. H.; Crossley, F. R. E. 1966: Structural permutations of flexible link mechanisms. ASME Paper No. 66-MECH-5.

(2) Her, I.; Midha, A. 1987: A compliance number concept for compliant mechanisms, and type synthesis. J. Mechanisms, Transmissions, and Automation in Design, Trans. ASME 109, 348-355.

(3) Ananthasuresh, G. K.; Kota, S.; Kikuchi N. 1994: Strategies for systematic synthesis of compliant MEMS. Proc. of the 1994 ASME Winter Annual Meeting, Chicago, Illinois DSC-55-2, 677-686.

(4) Sigmund, O. 1996: On the design of compliant mechanisms using topology optimization. Danish Center for Appl. Math. Mech. 535, 1-28.

(5) Nishiwaki, S.; Frecker, M. I.; Min, S.; Kikuchi, N. 1998: Topology optimization of compliant mechanisms using the homogenization method. Int. J. Numer. Meth. Eng. 42, 535-650.

(6) Onitsuka, K.,; Dogan, A.; Tressler, J. F.; Xu, Q.; Yoshikawa, S.; Newnham, R. E. 1995: Metal-ceramic composite transducer, the "Moonie". J. Intel. Mat. Sys. Struct. 6, 447-455.

(7) Kagawa, Y.; Tsuchiya, T.; Kataoka T. 1996: Finite element simulation of dynamic response of piezoelectric actuators. J. Sound and Vibration 191, 519-538.
(8) Bendsøe, M.P.; Kikuchi, N. 1988: Generating optimal topologies in structural design using a homogenization method. Comput. Methods. Appl. Mech. Engrg. 71, 197-224.

(9) Shield, R. T.; Prager, W. 1970: Optimal structural design for given deflection. J. Appl. Math. Phys., ZAMP 21, 513-523.

(10)Huang, N. 1971: On principle of stationary mutual complementary energy and its application to optimal structural design. J. Appl. Math. Phys., ZAMP 22, 609-620. 